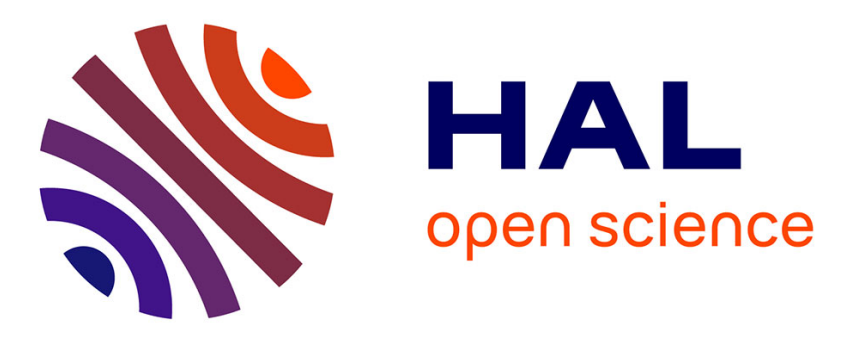

\title{
A fuzzy recognition model based on human skill integration
}

Cyril Mazaud, Vincent Bombardier, Pascal Lhoste, Raphaël Vogrig

\section{To cite this version:}

Cyril Mazaud, Vincent Bombardier, Pascal Lhoste, Raphaël Vogrig. A fuzzy recognition model based on human skill integration. 9th IFAC Symposium on Automated Systems Based on Human Skill and Knowledge, ASBhS'06, May 2006, Nancy, France. pp.CDROM. hal-00087466

\section{HAL Id: hal-00087466 https://hal.science/hal-00087466}

Submitted on 29 Sep 2006

HAL is a multi-disciplinary open access archive for the deposit and dissemination of scientific research documents, whether they are published or not. The documents may come from teaching and research institutions in France or abroad, or from public or private research centers.
L'archive ouverte pluridisciplinaire HAL, est destinée au dépôt et à la diffusion de documents scientifiques de niveau recherche, publiés ou non, émanant des établissements d'enseignement et de recherche français ou étrangers, des laboratoires publics ou privés. 


\title{
A FUZZY RECOGNITION MODEL BASED ON HUMAN SKILL INTEGRATION
}

\author{
Cyril Mazaud ${ }^{1,2}$, Vincent Bombardier ${ }^{1}$, Pascal Lhoste ${ }^{3}$, Raphaël Vogrig ${ }^{2}$ \\ ${ }^{1}$ Centre de Recherche en Automatique de Nancy (CRAN), CNRS UMR n'7039, Faculté des Sciences \\ Bd des Aiguillettes - BP 239 - 54506 Vandoeuvre-lès-Nancy Cedex, France \\ ${ }^{2}$ LuxScan Technologies, ZARE Ouest - L 4384 Ehlerange - Luxembourg \\ ${ }^{3}$ Equipe de Recherche sur les Processus Innovatifs (ERPI), EA n`3767, ENSGSI, Institut National \\ Polytechnique de Lorraine (INPL), 8 rue Bastien Lepage - BP 647 - 54010 NANCY Cedex, France
}

\begin{abstract}
This article presents the improvement of a defect recognition system for fibrous products by using knowledge integration from two expert fields. These two kinds of knowledge that we want to integrate respectively concern wood expertise and industrial vision expertise. First, extraction, modelling and integration of knowledge use the Natural language Information Analysis Method (NIAM) to be formalised from their natural language expression. Then, to improve a classical industrial recognition system using vision, we propose to use the resulting symbolic model of knowledge to partially build a numeric model of defect recognition. This model is created according to a tree structure where each inference engine is a Fuzzy Rules based Inference System. The expert knowledge model previously obtained is used to configure each node of the resulting hierarchical structure. The practical results we obtained with industrial data show the efficiency of such an approach. Copyright (C) 2006 IFAC
\end{abstract}

Keywords: Image processing, Pattern recognition, Fuzzy Inference, Knowledge representation.

\section{INTRODUCTION}

The increase of Information level that we can obtain on a system is essential for the improvement and the control of this system as well as processes linked in relation with it (automation, maintenance, relationship with the environment...). Two main sources allow to access to this part of Information: from human expert knowledge (expert in process, in products, in standards...) who provides rather qualitative a piece of Information on the studied system; and from data acquisition directly trough the system, which gives a rather quantitative piece of Information provided in the form of measures. Linking these two sources which are as different as complementary must contribute to a more complete and coherent information modelling, allowing a stronger integration of the processes making up the system. In this sense, the global framework of this project is to define a modelling and an integrating method, which allows on the one hand to transform Knowledge into piece of Information, on the other hand to extract the piece of Information from numerical data measured on the system and finally to integrate this Information of different origins in a same cognitive Information referential system.

This method is applied to the field of pattern recognition and, more precisely, to defects identification on wooden boards by an industrial vision system. It could be generalized to the whole sectorial fields where qualitative expert knowledge and quantitative data exist in parallel for the description of a same object.

\section{FRAMEWORK}

The work described here has been motivated by a collaborative effort between academia and industry. 
The Automatic Research Center of Nancy (CRAN) is the academic partner and Luxscan Technologies, a company based in the Luxembourg is the industrial partner. They concern the development of a recognition vision system for defects identification on wooden boards. Defect characterization forms a piece of information which is then utilized to estimate the wooden board quality with a view to cut it or to sort it. It is on the production line that board analysis is done, in real time. The figure (Fig. 1) illustrates the vision system principle.

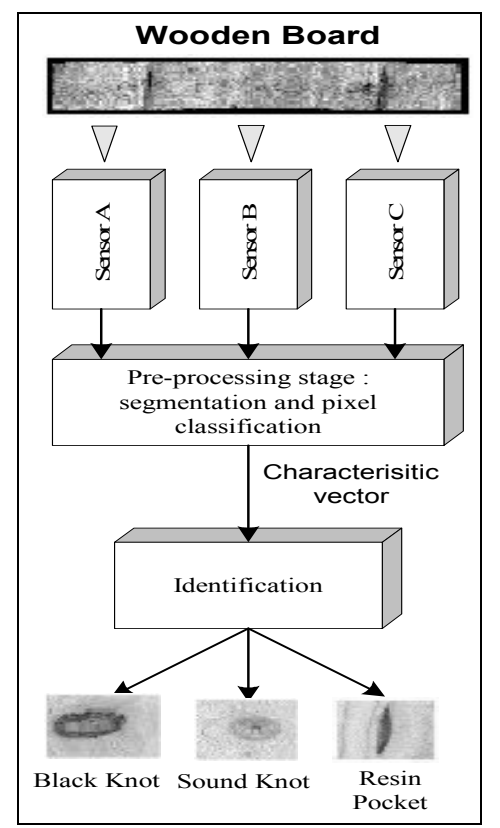

Fig. 1. Vision system principle.

The industrial vision system used is made up of two types of linear sensors (colour cameras and laser sources) which could be used in a combined way or in an independent way to acquire images of the wooden boards. The signals delivered by these sensors are then pre-processed and labelled to provide a piece of information with six classes of pixels. The classification is done either by a Bayesian classifier or by a threshold classifier, which parameters are set empirically. The segmentation realized from the labelled image, consists in aggregating close pixels, which do not belong to the sound wood class pixel. This part allows extracting the "defective" regions from "sound" regions. The detection or the nondetection of a defect belongs entirely to the segmenting stage. A set of parameters (surface, colour, orientation ...) is calculated on these defective regions to provide a characteristic vector used by the identification stage. The purpose of this stage is to name the defect: black knot, resin pocket, crack...

\section{PROPOSED METHOD}

In the field of systems which use human expertise and vision expertise, the main tendency resides in the building of a system based on heuristic rules (Foo, 1996) (Wang and Gong, 1998) (Wen and Tao, 1999) which describe how the decisional system works. However, even if the set of rules seems to work, none precision is given on their validation compared to the initial knowledge given by the expert. They are thus only a-priori informal processes of validation. To avoid encountering this problem, we propose to use an approach based on a formal validation based on the N.I.A.M. method (Habrias, 1998).

In the wood recognition field, the main processes generally use supervised or unsupervised neural networks to identify the different defects (Kauppinen, et al., 1999) (Lampinen, et al., 1995) (Pham and Sagiroglu, 2001). Even though the results obtained in these articles seem to satisfy the author, we choose not to use neural network methods because of their lack of interpretability concerning the structure of the layers generated during the learning stage. It is impossible to check the adequacy of the structure with the expert knowledge supposed to be modelled. At this level, the use of a method using tools from fuzzy sets (Zadeh, 1965) and more precisely from fuzzy logic (Bouchon-Meunier, 1995) could be justified by three main reasons. Firstly, defects classes are intrinsically fuzzy, there is not always a strict boundary between sound wood and defects; this transition is gradual. Secondly, defect classes or colour classes in output are not all disjointed. Indeed, the limit between a "small knot" and a "large knot" is not always strict. In practice, the defect classes are selected by the user in function of the defects which must be recognized in the handled wood specie. Thirdly, linguistic terms used by the experts to describe the defects (a knot rather round...) are often vague and imprecise. We represent these terms in the form of fuzzy terms characterized by their membership functions. These terms are thus computed with a rule-based mechanism.

In our global process, two main orientations are chosen to bring closer exploitable information for defects identification by vision: those which come from the vision system and those which come from the wood expert. On the one hand, we have numerical data in the form of a characteristic vector, which characterize the defect to identify through a relation that we call "numeric model". We choose to implement this model thanks to a system based on fuzzy linguistic rules (Dubois D. and H. Prade, 1992) in order to facilitate the interpretation of the identification mechanism. On the other hand, we have knowledge of defects which are expressed in natural language by the field expert in professional words. We formalize this knowledge in the form of what we call "symbolic" models with the NIAM method. To illustrate in a simple way our modelling and integrating thought process, we will only consider « knots » defects type, that is to say black knots, ring knots and sound knots.

The interest of this modelling and integration is to determine in a non-empiric way the more efficient parameters in input of each node of the identification module in relation with the outputs. In other words, choose the optimal characteristic vector for each inference engine. Figure (Fig. 2) shows our mining process. 


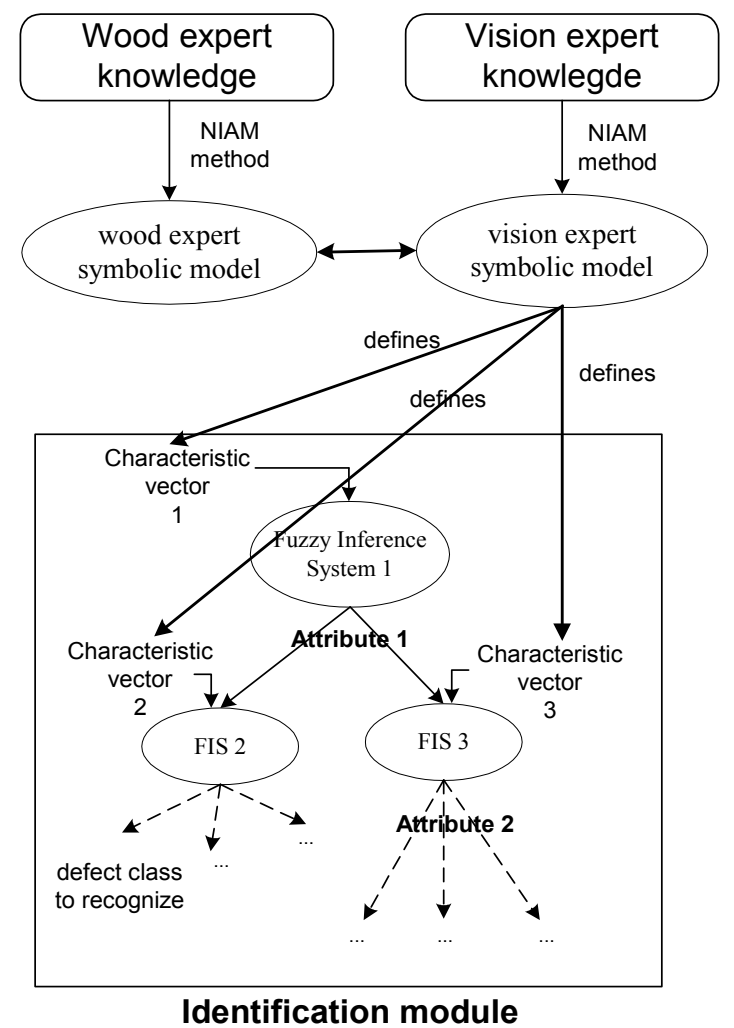

Fig. 2. Principle of the proposed expert knowledge integration method.

\section{EXPERT KNOWLEDGE MODELLING}

The symbolic model concerns expert knowledge formalization in the wood field and in the defects identification process field (vision system). The analysis method retained to elaborate this model is the N.I.A.M. (Nijssen or Natural Information Analysis Method) (Habrias, 1998). We also use one of its derived formalism, O.R.M. (Object Role Modelling) (Halpin, 2001; Halpin, 1998) to represent the results of this analysis.

\subsection{NIAM method and ORM formalism.}

The NIAM method relies on a linguistic text analysis. From the expression of observable facts implicating objects, this method allows to distinguish non-lexical objects (NOLOTs) and lexical objects (LOTs) and the facts which link them together. The figure (Fig. 3) illustrates some syntactical elements of the NIAM/ORM formalism allowing representing these objects and their relations.

For more information concerning the NIAM method and the proposed NIAM/ORM formalism, the reader can refer itself to (Halpin, 1998).

This method has a significant interest because it relies directly on an expression of facts stated in natural language. The resulting information model could also be submitted to validation by the expert under a comprehensive form, i.e. in Binary Natural Language (BNL) which is a transcription of the information model also called "paraphrase action".

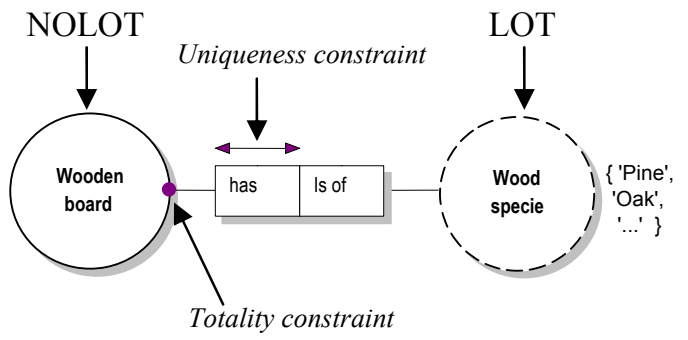

Each Wooden bord has one and only one Wood Specie.

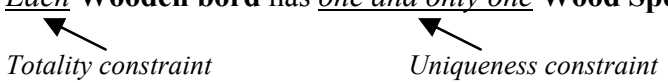

$\underline{A}$ Wood Specie is of one or several Wooden Boards.
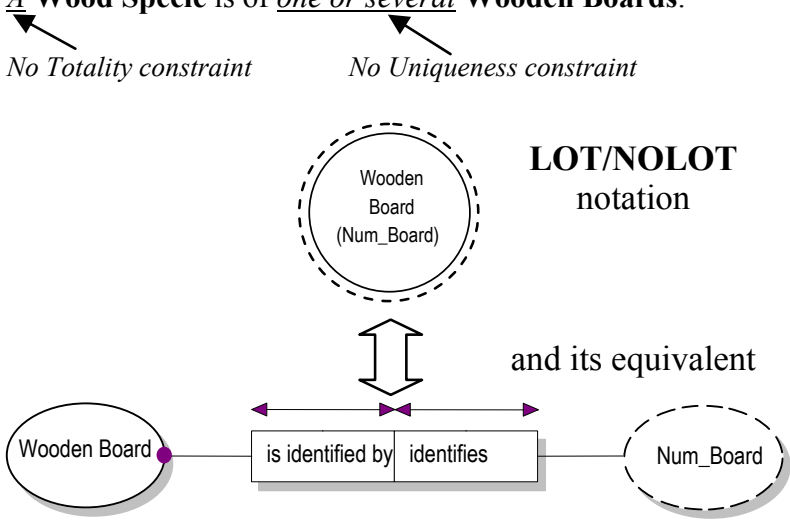

Fig. 3. Elements of NIAM/ORM formalism.

The method allows completing progressively the model by asking to the expert precise questions induced by the method, also in natural language (for example: does a defect have one or several colours?). We thus obtain a model completed by totality and uniqueness constraints.

\subsection{Wood field knowledge modelling.}

To create this model, we ask the wood field expert to write a list, as complete as possible and in a natural language form, of the different defects that can be found in the species handled. This expression is sufficient to recognize in naked eye any kind of defect in a wood sample.

The figure (Fig. 4) represents the generic model for "knots" defect type including totality and uniqueness constraints characterizing a generic modelling.

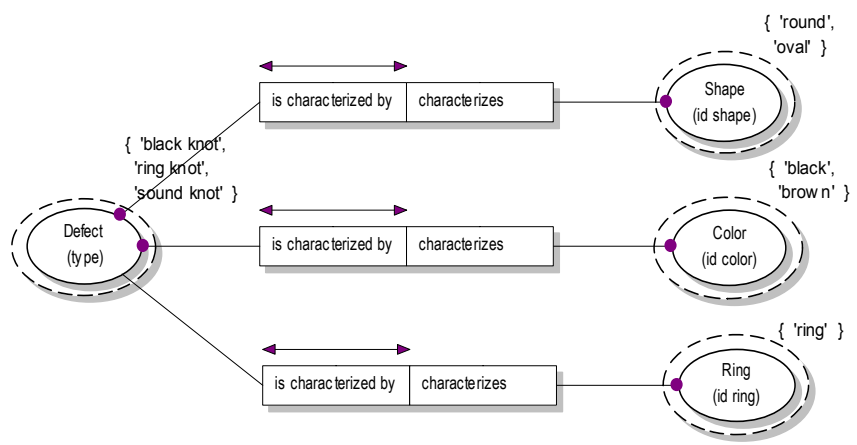

Fig. 4. Generic model of the "knots" defect type. 
In order to get this model validated by the expert who expressed the knowledge used for its development, we transcribe it in Binary Natural Language. The result of this transcription is thus a text that is closed to natural language which does not impose to the expert any particular knowledge about the modelling method. Here is the text submitted to the expert for validation:

Each Defect is characterized by one and only one Shape. Each Shape characterizes one or several Defects. Each Defect is characterized by one and only one Colour. Each Colour characterizes one or several Defects. A Defect is characterized by one and only one Ring. Each Ring characterizes one or several Defects.

Notice here that the sentence " $A$ Defect is characterized by one and only one Ring" does not indicate that all defects have a ring. It just means that a defect can have a ring but is not forced to have one.

\subsection{Vision field knowledge modelling.}

Just like for "wood expert" symbolic model, we register the vision expert knowledge. This knowledge is about the defects identification system and more precisely on the parameters useful to quantify the characteristics of the defect to recognize. We apply the same principle than the one used for the wood expert symbolic model. We thus obtain a characterization of all the defects encountered, but this time, in a vision parameters point of view.

The figure (Fig. 5) represents a partial formalization of the colour concept with a vision point of view.

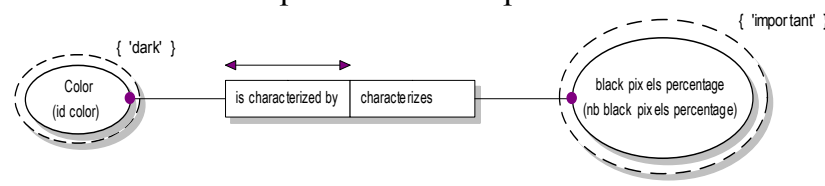

Fig. 5. Partial NIAM/ORM formalization of colour parameter.

This modelling represents the following fact, by considering that a colour is defined by other parameters than black pixels number: a precise colour is characterized by a unique black pixels number. But the opposite is not true. To a given black pixels number can correspond several colours.

We do the same for Shape and Ring characteristics. Notice that for confidential reasons we could not expose entirely the expert knowledge models. That is why just only some of the used parameters for each characteristic will be revealed.

\section{NUMERIC MODEL}

To link the symbolic field and the numeric field, we use a method based on fuzzy subsets which allows supporting qualitative reasoning (Zadeh, 1992). This process is essential to compare expert information (in natural language) with the response of the system (fuzzy data). Our numeric model relies on a series of fuzzy inferences system which have common structural characteristics.

In this perspective of linking symbolic and numeric fields, we noticed an interesting work led by (Galichet and Foulloy, 2003) in which they propose different architectures to "combine conventional regulators and knowledge-based procedures in a unified control structure". In other words, they proposed a framework allowing achieving "a collaboration between numeric and expert processing" implemented with fuzzy linguistic systems.

The method we use is an iterative method (Ishibuchi, et al., 1994) with a supervised learning step from a sample data set. The algorithm implements a mechanism of supervised teaching. It generates fuzzy rules like "If... Then..." which define the defects perception which the system has. The expert must prepare samples defects set to generate the fuzzy rules thanks to the algorithm.

If we have two characteristics in input $\left(\mathrm{V}_{1}\right.$ and $\left.\mathrm{V}_{2}\right)$ and one output $\left(Z_{3}\right)$, the general form of the fuzzy rule is:

IF $V_{1}$ is $A_{i}$ AND IF $V_{2}$ is $A_{j}$ THEN $Z_{3}$ is in the defect class $C_{k}$

$\mathrm{V}_{1}$ and $\mathrm{V}_{2}$ : inputs data (intensity and surface by example); $\mathrm{Z}_{3}$ : output data (defect name); $\mathrm{A}_{\mathrm{i}}$ and $\mathrm{A}_{\mathrm{j} \text { : }}$ linguistic terms representing fuzzy subsets ("light" and "dark" for example); $\mathrm{C}_{\mathrm{k}}$ : class of $\mathrm{k}^{\text {th }}$ defect.

Notice that $A_{i}$ and $A_{j}$ are linguistic terms which qualify a parameter. This qualification is called the fuzzification (Bouchon-Meunier, 1995) which allows to split the representation space of the parameter. This splitting can be empirically done (case of an equal distribution of the representation space) or guided by the expert (case of a non-equal distribution). The rules compounding our rule basis are conjunctive rules and the inference engine is build on the Larsen model (Bombardier, et al., 2004).

\section{EXPERT KNOWLEDGE INTEGRATION}

\subsection{Knowledge integration.}

The expert knowledge integration in the numeric model, which is the centre of data processing, can only be done by putting in relation expert knowledge from the two fields of competence, i.e. wood field and industrial vision field.

The mechanism which allows linking the two fields consists in putting together the symbolic models of wood expertise and vision expertise. The result of this connection is a new symbolic model illustrated by the figure (Fig. 6). Notice that for confidential reasons, we do not expose the parameters used. Thanks to this model, we can choose the best parameters for the characteristic vector which allows identifying the three defects chosen in example. For these three defect types, the common characteristic being the shape (as they are knots, their shape is then similar), only the characteristics of colour and ring are discriminating in their identification. 


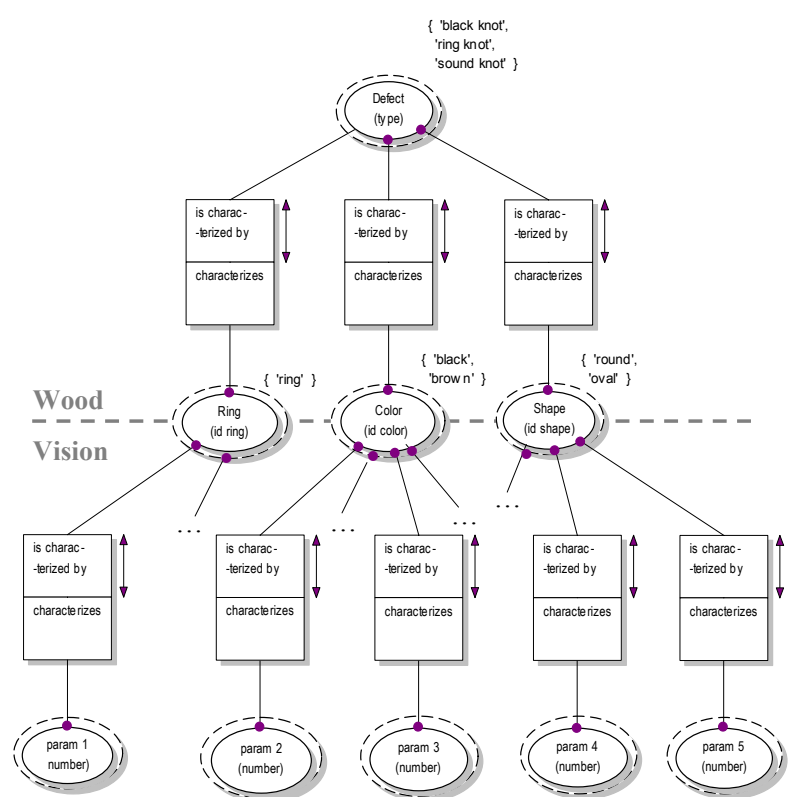

Fig. 6. Knowledge integration process.

The characteristic of shape allows identifying rather round defects from rather elongated defects. This characteristic is utilized upstream in the model. Notice here that we tackle the notion of hierarchy between defects attributes. This knowledge is not yet formalized neither modelled; by consequent, at present, we utilize an a priori knowledge of this hierarchy to build the decisional structure of our numeric model. The figure (Fig. 7) represents a part of numeric model and more precisely the inference engine identifying the three defects.

The interest of this integration by a Fuzzy Inferences System based on a fuzzy linguistic rules system resides in interpreting linguistically each inference of the model. It is thus possible to understand the numeric model behaviour and check, on the one hand, that expert knowledge was well integrated in the model, and on the other hand, that there is a coherence between data presented to the model and knowledge utilized to build it. A difference between data and knowledge can mean that data utilized are not really representative of the field or that expert knowledge expressed initially is not sufficient or incomplete.

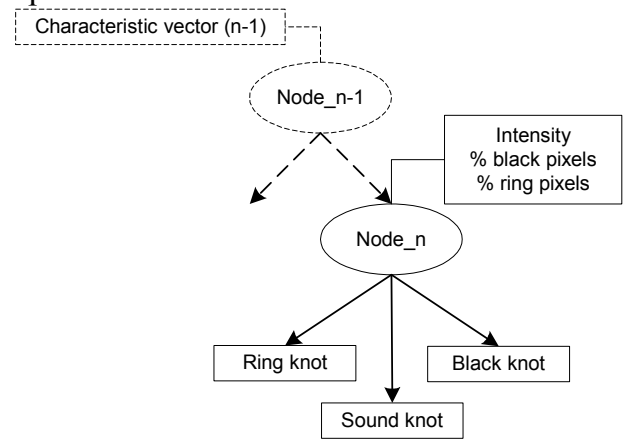

Fig. 8. Results of the expert knowledge integration for the choice of the characteristic vector of the node "Node_n".

\subsection{Results.}

We build three types of numeric models:
- as a reference model, we use the Point method which is utilized by the LuxScan Technologies Company,

- a Single Inference (model SI) which not takes into account expert knowledge,

- an Arborescent Inference Structure (model AIS) with expert knowledge integration to take into account the industrial time processing constraint.

Moreover, we test different two types of fuzzification to see its influence on the recognition rate.

An equal distributed fuzzification with an equal splitting of the representation space of each variable in 3 terms.

A non-equal distributed fuzzification with a splitting in 2 or 3 terms according to expert knowledge.

The table (Tab. 1) summarizes the results obtained with two methods; the Point method currently used by the industrial and the Fuzzy method developed. Theses tests were led on a 327 defects sample provided by the LuxScan Technologies Company.

Table 1 Results obtained

\begin{tabular}{lc} 
& Identification rate \\
\hline Point method & $68.8 \%$ \\
Fuzzy method & \\
model SI with equal distribution & $69.7 \%$ \\
model AIS with equal distribution & $73.7 \%$ \\
model SI with non-equal distribution & $77.4 \%$ \\
model AIS with non-equal distribution & $82.6 \%$ \\
\hline
\end{tabular}

The main conclusions from these tests are the following. Firstly, in term of identification rate, the fuzzy method gives better results than the Point method. In all cases tested, the worst result given by the fuzzy method is better than the Point method.

Secondly, by only considering the results given for the different fuzzifications and the different models, we can see that the better result is given with an arborescent model with a representation space cutting done in function of expert knowledge (what we call a non-equal distribution). This result confirms our choice of using and integrating expert knowledge to build our numeric model and to choose the number of fuzzification terms of each parameter.

\section{CONCLUSION AND PERSPECTIVES}

The knowledge integration we propose allows a better coherence between numeric model of the data processing and expert knowledge used to build it. This integration also allows the obtaining of a non empiric model in the sense that each parameter forming each decisional node are not yet chosen empirically but chosen from knowledge given by the experts of the two studied fields. The results here presented are obtained from a module called Fuzzy Approximate Classifier that we developed to configure, create and test our numeric model. As said before in this article, it works according a Fuzzy Linguistic Rules based system. The results obtained thanks to expert knowledge integration in our numeric model are encouraging. Indeed, they 
demonstrate that a link exists between human expertise, represented by symbolic models, and its numeric equivalent. However, with a better modelling technique which takes into account the inherent vagueness of cognitive knowledge expressed in natural language, we could improve its integration and by itself improves the identification results. That is why we propose to work again on M. Zgorzelski and Z. Zalewski study on Fuzzy NIAM (Zgorzelski and Zalewski, 1996) to provide a unique frame, on the one hand to expert knowledge modelling and integration (qualitative aspect) which is certain but inaccurate, and on the other hand to measured data (quantitative aspect) which is accurate but uncertain. We also want to enhance the links between the symbolic and the numeric models by using fuzzy constraints (particularization of procedural constraints in ORM) in our symbolic model to show that rules expressed by the expert and formalized in our symbolic model are necessarily in the rule basis of our numeric model.

Future works aims at acquiring and formalizing knowledge not yet modelled. This knowledge concerns hierarchy between defects attributes, that is to say the attributes order of importance to identify a defect. The expert will consider the colour, the shape, the size ... as first discriminating characteristic? This knowledge could allow us refining the present numeric model in the sense that we will not have an empirical arborescent structure but an arborescent which could be qualified as structured. We would also like to formalize expert knowledge concerning the fusion between defects that could refine the gross result delivered by the identification module by maintaining or refuting this result. Indeed, knowledge on the considered defect's environment will refine the final decision delivered by the system. By environment, we consider either the direct environment of the defect by the analysis of its neighbourhood (only considering the current side of the board), or the indirect environment by considering all the sides of the board. To finish, we would also like to work on the fuzzification stage and more particularly developing an adapted fuzzification which will take in account expert knowledge but also data provided by the learning sample. In fact, the principle will rely on cooperation between the symbolic and the numeric world; the symbolic world will provide the basic number of fuzzification terms for each parameter and the numeric world will modify the representation space in function of its analysis.

\section{ACKNOWLEDGMENTS}

The authors wish to thank their industrial partner the LuxScan Technologies Company, their staff and especially M. Jean-Yves Hergott who is responsible for projects development in the company and who follows the present works.

\section{REFERENCES}

Bombardier, V., C. Mazaud, and P. Lhoste (2004). Modélisation et intégration de connaissances métier pour l'identification de défauts par règles linguistiques floues. TS Traitement $d u$ Signal, vol. 31, 227-247.

Bouchon-Meunier, B. (1995). La logique floue et ses applications. Addison-Wesley.

Dubois, D. and H. Prade (1992). Fuzzy rules in knowledge-based systems - Modelling gradedness, uncertainty and preference. An introduction to fuzzy logic application in intelligent systems. Kluwer-Dordrecht, 45-68.

Foo, SY. (1996). A rule-based machine vision for fire detection in aircraft dry bays and engine compartments. Knowledge-Based Systems, vol. 9, 531-540.

Galichet, S., and L. Foulloy (2003). Integrating expert knowledge into industrial control structures. Computer in Industry, vol. 52, 235-251.

Habrias, H. (1998). Le modèle relationnel binaire. Méthode I.A. (NIAM). Editions Eyrolles.

Halpin, T.A. (2001). Information Modelling and Relational Databases. Morgan Kaufman edition.

Halpin, T.A. (1998). Object Role Modeling (ORM/NIAM). Handbook on Architectures of Information Systems.

Ishibuchi, H., K. Nozaki, N. Yamamoto, and H. Tanaka (1994). Construction of fuzzy classification systems with rectangular fuzzy rules using genetic algorithms. Fuzzy sets and systems, vol. 65, 237-253.

Kaufmann, A. (1975). Introduction à la théorie des sous-ensembles flous. Edition Masson, 1975.

Kauppinen, H., H. Rautio and O. Silven (1999). Nonsegmenting defect detection and SOM based classification for surface inspection using color vision. Polarization and Color Techniques in Industrial Inspection (SPIE 3826), 270-280.

Lampinen, J., S. Smolander and M. Korhonen (1995). Wood surface inspection system based on generic visual features. International Conference on Artificial Neural Networks ICANN'95.

Pham, D.T. and S. Sagiroglu (2001). Training multilayered perceptrons for pattern recognition: a comparative study of four training algorithms. International Journal of Machine Tools \& Manufacture, vol. 41, 419-430.

Wen, Z. and Y. Tao (1999). Building a rule-based machine-vision system for defect inspection on apple sorting and packing lines. Expert Systems with Applications, vol. 16, 307-313.

Wang, C.S. and X.K. Gong (1998). A fuzzy approximate reasoning model for a rule-based system in laser threat recognition. Fuzzy Sets and System, vol. 96, 139-146.

Zadeh, L.A. (1992). The calculus of fuzzy if/then rules. AI Expert, vol. 7, 23-27.

Zadeh, L.A. (1965). Fuzzy sets. Information and control, vol. 8, 338-353.

Zgorzelski, M. and Z. Zalewski (1996). Fuzzy NIAM for real world data modeling. I.S.A.T.A. $29^{\text {th }}$ International Symposium on Automative Technology and Automation. 